\title{
Green's Function Method for Self-Adjoint Realization of Boundary-Value Problems with Interior Singularities
}

\author{
K. Aydemir and O. Sh. Mukhtarov \\ Department of Mathematics, Faculty of Arts and Science, Gaziosmanpaşa University, 60250 Tokat, Turkey \\ Correspondence should be addressed to K. Aydemir; kadriye.aydemir@gop.edu.tr
}

Received 24 June 2013; Accepted 5 September 2013

Academic Editor: Ravshan Ashurov

Copyright (C) 2013 K. Aydemir and O. Sh. Mukhtarov. This is an open access article distributed under the Creative Commons Attribution License, which permits unrestricted use, distribution, and reproduction in any medium, provided the original work is properly cited.

\begin{abstract}
The purpose of this paper is to investigate some spectral properties of Sturm-Liouville type problems with interior singularities. Some of the mathematical aspects necessary for developing our own technique are presented. By applying this technique we construct some special solutions of the homogeneous equation and present a formula and the existence conditions of Green's function. Furthermore, based on these results and introducing operator treatment in adequate Hilbert space, we derive the resolvent operator and prove self-adjointness of the considered problem.
\end{abstract}

\section{Introduction}

For inhomogeneous linear systems, the basic superposition principle says that the response to a combination of external forces is the self-same combination of responses to the individual forces. In a finite-dimensional system, any forcing function can be decomposed into a linear combination of unit impulse forces, each applied to a single component of the system, and so the full solution can be written as a linear combination of the solutions to the impulse problems. This simple idea will be adapted to boundary value problems governed by differential equations, where the response of the system to a concentrated impulse force is known as Green's function. With Green's function in hand, the solution to the inhomogeneous system with a general forcing function can be reconstructed by superimposing the effects of suitably scaled impulses. Green's function method provides a powerful tool to solve linear problems consisting of a differential equation (partial or ordinary, with, possibly, an inhomogeneous term) and enough initial and/or boundary conditions (also possibly inhomogeneous) so that this problem has a unique solution. The history of Green's function dates back to 1828, when Green [1] published work in which he sought solutions of Poisson's equation $\nabla^{2} u=f$ for the electric potential $u$ defined inside a bounded volume with specified boundary conditions on the surface of the volume. He introduced a function now identified as what Riemann later coined Green's function. In 1877, Neumann [2] embraced the concept of Green's function in his study of Laplace's equation, particularly in the plane. He found that the two-dimensional equivalent of Green's function was not described by singularity of the form $1 /\left|r-r_{0}\right|$ as in the three-dimensional case but by a singularity of the form $\log \left(1 /\left|r-r_{0}\right|\right)$. With the function's success in solving Laplace's equation, other equations began to be solved using Green's function. The heat equation and Green's function have a long association with each other. After discussing heat conduction in free space, the classic solutions of the heat equation in rectangular, cylindrical, and spherical coordinates are offered. In the case of the heat equation, Hobson [3] derived the freespace Green's function for one, two and three dimensions, and the French mathematician Appell [4] recognized that there was a formula similar to Green's for the one-dimensional heat equation. Green's function is particularly well suited for wave problems with the detailed analysis of electromagnetic waves in surface wave guides and water waves. The leading figure in the development of Green's function for the wave equation was Kirchhoff [5], who used it during his study 
of the three-dimensional wave. Starting with Green's second formula, he was able to show that the three-dimensional Green's function is

$$
g(x, y, z, t \mid \xi, \eta, \varsigma, \tau)=\frac{\delta(t-\tau-R / c)}{4 \pi R},
$$

where $R=\sqrt{(x-\xi)^{2}+(y-\eta)^{2}+(z-\varsigma)^{2}}$.

The application of Green's function to ordinary differential equations involving boundary-value problems began with the work of Burkhardt [6]. Determination of Green's function is also possible using Sturm-Liouville theory. This leads to series representation of Green's function. SturmLiouville problems which contained spectral parameter in boundary conditions form an important part of the spectral theory of boundary value problems. This type of problems has a lot of applications in mechanics and physics (see [79] and references cited therein). In the recent years, there has been increasing interest in this kind of problems which also may have discontinuities in the solution or its derivative at interior points (see [10-18]). In this study, we will investigate some spectral properties of the Sturm-Liouville differential equation on two intervals:

$$
\begin{array}{r}
\mathscr{L} y:=-y^{\prime \prime}(x)+q(x) y(x)=\lambda y(x), \\
x \in[a, c) \cup(c, b]
\end{array}
$$

on $[a, c) \cup(c, b]$, with eigenparameter-dependent boundary conditions at the end points $x=a$ and $x=b$. One has,

$$
\begin{gathered}
\tau_{1}(y):=\alpha_{10} y(a)+\alpha_{11} y^{\prime}(a)=0 \\
\tau_{2}(y):=\alpha_{20} y(b)-\alpha_{21} y^{\prime}(b)+\lambda\left(\alpha_{20}^{\prime} y(b)-\alpha_{21}^{\prime} y^{\prime}(b)\right)=0
\end{gathered}
$$

and the transmission conditions at the singular interior point $x=c$

$$
\begin{aligned}
\tau_{3}(y):= & \beta_{11}^{-} y^{\prime}(c-)+\beta_{10}^{-} y(c-) \\
& +\beta_{11}^{+} y^{\prime}(c+)+\beta_{10}^{+} y(c+)=0, \\
\tau_{4}(y):= & \beta_{21}^{-} y^{\prime}(c-)+\beta_{20}^{-} y(c-) \\
& +\beta_{21}^{+} y^{\prime}(c+)+\beta_{20}^{+} y(c+)=0,
\end{aligned}
$$

where the potential $q(x)$ is real continuous function in each of the intervals $[a, c)$ and $(c, b]$ and has finite limits $q(c \mp 0)$, $\lambda$ is a complex spectral parameter, $\alpha_{i j}, \beta_{i j}^{ \pm}, \quad(i=1,2$ and $j=$ $0,1)$, and $\alpha_{i j}^{\prime}(i=2$ and $j=0,1)$ are real numbers.

Our problem differs from the usual regular SturmLiouville problem in the sense that the eigenvalue parameter $\lambda$ is contained in both differential equation and boundary conditions, and two supplementary transmission conditions at one interior point are added to boundary conditions. Such problems are connected with discontinuous material properties, such as heat and mass transfer, vibrating string problems when the string loaded additionally with points masses, diffraction problems $[8,9]$, and varied assortment of physical transfer problems. We develop our own technique for the investigation of some spectral properties of this problem. In particular, we construct the Green's function and adequate Hilbert space for self-adjoint realization of the considered problem.

\section{Some Basic Solutions and Green's Function}

Denote the determinant of the $k$ th and $j$ th columns of the matrix

$$
T=\left[\begin{array}{llll}
\beta_{10}^{+} & \beta_{11}^{+} & \beta_{10}^{-} & \beta_{11}^{-} \\
\beta_{20}^{+} & \beta_{21}^{+} & \beta_{20}^{-} & \beta_{21}^{-}
\end{array}\right]
$$

by $\Delta_{k j}(1 \leq k<j \leq 4)$. For self-adjoint realization in adequate Hilbert space, everywhere below we will assume that

$$
\Delta_{12}>0, \quad \Delta_{34}>0 .
$$

With a view to construct the Green's function we will define two special solutions of (2) by our own technique as follows. At first, consider the next initial-value problem on the left interval $[a, c)$

$$
\begin{gathered}
-y^{\prime \prime}+q(x) y=\lambda y, \\
y(a)=\alpha_{11}, \quad y^{\prime}(a)=-\alpha_{10} .
\end{gathered}
$$

It is known that this problem has an unique solution $u=$ $\varphi^{-}(x, \lambda)$ which is an entire function of $\lambda \in \mathbb{C}$ for each fixed $x \in[a, c)$ (see, e.g., [19]). By applying the similar method of [13], we can prove that (2) on the right interval $(c, b]$ has an unique solution $u=\varphi^{+}(x, \lambda)$ satisfying the equalities

$$
\begin{aligned}
& \varphi^{+}(c+, \lambda)=\frac{1}{\Delta_{12}}\left(\Delta_{23} \varphi^{-}(c-, \lambda)+\Delta_{24} \frac{\partial \varphi^{-}(c-, \lambda)}{\partial x}\right), \\
& \frac{\partial \varphi^{+}(c+, \lambda)}{\partial x}=\frac{-1}{\Delta_{12}}\left(\Delta_{13} \varphi^{-}(c-, \lambda)+\Delta_{14} \frac{\partial \varphi^{-}(c-, \lambda)}{\partial x}\right),
\end{aligned}
$$

which is also an entire function of the parameter $\lambda$ for each fixed $x \in[c, b]$. Consequently, the solution $u=\varphi(x, \lambda)$ defined by

$$
\varphi(x, \lambda)= \begin{cases}\varphi^{-}(x, \lambda), & x \in[a, c) \\ \varphi^{+}(x, \lambda), & x \in(c, b]\end{cases}
$$

satisfies (2) on whole $[a, c) \cup(c, b]$, the first boundary condition of (3), and both transmission conditions (5).

By the same technique, we can define the solution by

$$
\psi(x, \lambda)= \begin{cases}\psi^{-}(x, \lambda), & x \in[a, c) \\ \psi^{+}(x, \lambda), & x \in(c, b]\end{cases}
$$


so that

$$
\begin{aligned}
& \psi^{+}(b, \lambda)=\alpha_{21}+\lambda \alpha_{21}^{\prime}, \quad \frac{\partial \psi^{+}(b, \lambda)}{\partial x}=\alpha_{20}+\lambda \alpha_{20}^{\prime}, \\
& \psi^{-}(c-, \lambda)=\frac{-1}{\Delta_{34}}\left(\Delta_{14} \psi^{+}(c+, \lambda)+\Delta_{24} \frac{\partial \psi^{+}(c+, \lambda)}{\partial x}\right), \\
& \frac{\partial \psi^{-}(c-, \lambda)}{\partial x}=\frac{1}{\Delta_{34}}\left(\Delta_{13} \psi^{+}(c+, \lambda)+\Delta_{23} \frac{\partial \psi^{+}(c+, \lambda)}{\partial x}\right) .
\end{aligned}
$$

Consequently, $\psi(x, \lambda)$ satisfies (2) on whole $[a, c) \cup(c, b]$, the second boundary condition (4), and both transmission condition (5). By using (9), (10), (14), and (15) and the well-known fact that the Wronskians $w^{-}(\lambda):=W\left[\varphi^{-}(x, \lambda), \psi^{-}(x, \lambda)\right]$ and $w^{+}(\lambda):=W\left[\varphi^{+}(x, \lambda), \psi^{+}(x, \lambda)\right]$ are independent of variable $x$, it is easy to show that $\Delta_{12} w^{+}(\lambda)=\Delta_{34} w^{-}(\lambda)$. We will introduce the characteristic function for the problems (2)-(5) as

$$
w(\lambda):=\Delta_{34} w^{-}(\lambda)=\Delta_{12} w^{+}(\lambda) .
$$

Similar to [13], we can prove that there are infinitely many eigenvalues $\lambda_{n}, n=1,2, \ldots$ of the BVTP (2)-(5) which coincide with the zeros of characteristic function $w(\lambda)$.

Now, let us consider the nonhomogenous differential equation

$$
y^{\prime \prime}+(\lambda-q(x)) y=f(x)
$$

on $[a, c) \cup(c, b]$ together with the same boundary and transmission conditions $(2)-(5)$, when $w(\lambda) \neq 0$. We will search the solution of this problem in the form (see, for example, [13]):

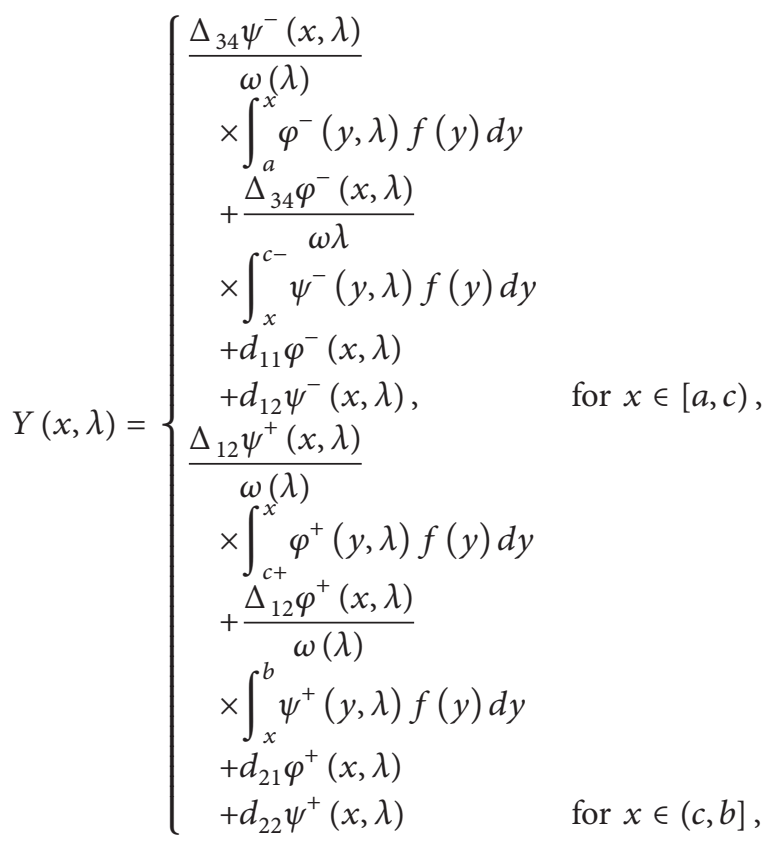

where $d_{i j}(i, j=1,2)$ are arbitrary constants. Putting in (3)(5) we have $d_{12}=0, d_{21}=0$,

$$
\begin{aligned}
& d_{11}=\frac{\Delta_{12}}{w(\lambda)} \int_{c+}^{b} f(y) \psi^{+}(y, \lambda) d y, \\
& d_{22}=\frac{\Delta_{34}}{w(\lambda)} \int_{a}^{c-} f(y) \varphi^{-}(y, \lambda) d y .
\end{aligned}
$$

Now, by substituting these equalities in (18), the following formula is obtained for the solution $Y=Y_{0}(x, \lambda)$ of (17) under boundary and transmission conditions (3)-(5):

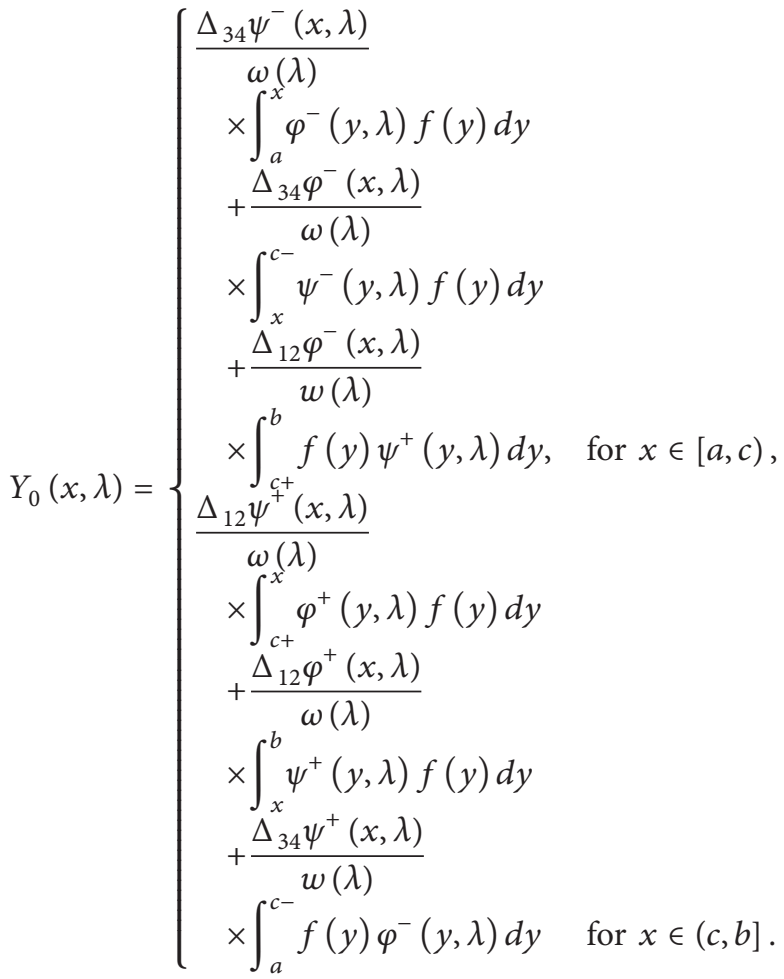

From this formula, we find that the Green's function of the problem (2)-(5) has the form:

$$
G_{0}(x, y ; \lambda)=\left\{\begin{array}{rr}
\frac{\varphi(y, \lambda) \psi(x, \lambda)}{\omega(\lambda)}, & \text { for } a \leq y \leq x \leq b, \\
x, y \neq c, \\
\frac{\varphi(x, \lambda) \psi(y, \lambda)}{\omega(\lambda)}, & \text { for } a \leq x \leq y \leq b, \\
x, y \neq c,
\end{array}\right.
$$

and the solution (20) can be rewritten in the terms of this Green's function as

$$
\begin{aligned}
Y_{0}(x, \lambda)= & \Delta_{34} \int_{a}^{c-} G_{0}(x, y ; \lambda) f(y) d y \\
& +\Delta_{12} \int_{c+}^{b} G_{0}(x, y ; \lambda) f(y) d y .
\end{aligned}
$$




\section{Construction of the Resolvent Operator by means of Green's Function in the Adequate Hilbert Space}

In this section, we define a linear operator $A$ in suitable Hilbert space in such a way that the considered problem can be interpreted as the eigenvalue problem of this operator. For this, we assume that $\Delta_{0}:=\alpha_{21} \alpha_{20}^{\prime}-\alpha_{20} \alpha_{21}^{\prime}>0$ and introduce a new inner product in the Hilbert space $H=\left(L_{2}[a, c) \oplus\right.$ $\left.L_{2}(c, b]\right) \oplus \mathbb{C}$ by

$$
\begin{aligned}
\langle F, G\rangle_{1}:= & \Delta_{34} \int_{a}^{c-} f(x) \overline{g(x)} d x \\
& +\Delta_{12} \int_{c+}^{b} f(x) \overline{g(x)} d x+\frac{\Delta_{12}}{\Delta_{0}} f_{1} \overline{g_{1}},
\end{aligned}
$$

for $F=\left(f(x), f_{1}\right), G=\left(g(x), g_{1}\right) \in H$.

Remark 1 . Note that this modified inner product is equivalent to standard inner product of $\left(L_{2}[a, c) \oplus L_{2}(c, b]\right) \oplus \mathbb{C}$; so $H_{1}=$ $\left(L_{2}[a, c) \oplus L_{2}(c, b] \oplus \mathbb{C},\langle\cdot, \cdot\rangle_{1}\right)$ is also Hilbert space.

For convenience, denote

$$
\begin{aligned}
& T_{b}(f):=\alpha_{20} f(b)-\alpha_{21} f^{\prime}(b), \\
& T_{b}^{\prime}(f):=\alpha_{20}^{\prime} f(b)-\alpha_{21}^{\prime} f^{\prime}(b),
\end{aligned}
$$

and define a linear operator

$$
A\left(\mathscr{L} f(x), T_{b}^{\prime}(f)\right)=\left(\mathscr{L} f,-T_{b}(f)\right)
$$

with the domain $D(A)$ consisting of all elements $\left(f(x), f_{1}\right) \epsilon$ $H_{1}$ such that $f(x)$ and $f^{\prime}(x)$ are absolutely continuous in each interval $[a, c)$ and $(c, b]$ and has a finite limit $f(c \mp 0)$ and $f_{1}^{\prime}(c \mp 0), \mathscr{L} f \in L_{2}[a, b], \tau_{1} f=\tau_{3} f=\tau_{4} f=0$ and $f_{1}=$ $T_{b}^{\prime}(f)$.

Consequently the problems (2)-(5) can be written in the operator form as

$$
\begin{gathered}
A F=\lambda F, \\
F=\left(f(x), T_{b}^{\prime}(f)\right) \in D(A)
\end{gathered}
$$

in the Hilbert space $H_{1}$. It is easy to see that the operator $A$ is well defined in $H_{1}$. Let $A$ be defined as above and let $\lambda$ not be an eigenvalue of this operator. For construction of the resolvent operator $R(\lambda, A):=(\lambda-A)^{-1}$, we will solve the operator equation

$$
(\lambda-A) Y=F
$$

for $F \in H_{1}$. This operator equation is equivalent to the nonhomogeneous differential equation

$$
y^{\prime \prime}+(\lambda-q(x)) y=f(x),
$$

on $[a, c) \cup(c, b]$ subject to nonhomogeneous boundary conditions and homogeneous transmission conditions

$$
\tau_{1}(y)=\tau_{3}(y)=\tau_{4}(y)=0, \quad \tau_{2}(y)=-f_{1} .
$$

Let $\operatorname{Im} \lambda \neq 0$. We already know that the general solution of (28) has the form (18). Putting this general solution in (29) yields

$$
\begin{gathered}
d_{11}=\frac{\Delta_{12}}{\omega(\lambda)} \int_{c+}^{b} \psi^{+}(y, \lambda) f(y) d y+\frac{\Delta_{12} f_{1}}{\omega(\lambda)}, \\
d_{12}=0, \quad d_{21}=\frac{\Delta_{12} f_{1}}{\omega(\lambda)}, \\
d_{22}=\frac{\Delta_{34}}{\omega(\lambda)} \int_{a}^{c-} \varphi^{-}(y, \lambda) f(y) d y .
\end{gathered}
$$

Thus, the problems (28)-(29) have a unique solution

$$
Y(x, \lambda)= \begin{cases}\frac{\Delta_{34} \psi^{-}(x, \lambda)}{\omega(\lambda)} \\ \quad \times \int_{a}^{x} \varphi^{-}(y, \lambda) f(y) d y \\ \quad+\frac{\Delta_{34} \varphi^{-}(x, \lambda)}{\omega(\lambda)} \\ \quad \times \int_{x}^{c^{-}} \psi^{-}(y, \lambda) f(y) d y \\ \quad+\frac{\Delta_{12} \varphi^{-}(x, \lambda)}{\omega(\lambda)} \\ \quad \times \int_{c+}^{b} \psi^{+}(y, \lambda) f(y) d y \\ \frac{\Delta_{12} \psi^{+}(x, \lambda)}{\omega(\lambda)} \\ \quad \times \int_{c+}^{x} \varphi^{+}(y, \lambda) f(y) d y \\ \quad+\frac{\Delta_{12} \varphi^{+}(x, \lambda)}{\omega(\lambda)} \\ \quad \times \int_{x}^{b} \psi^{+}(y, \lambda) f(y) d y \\ +\frac{\Delta_{34} \psi^{+}(x, \lambda)}{\omega(\lambda)} \\ \quad \times \int_{a}^{c-} \varphi^{-}(y, \lambda) f(y) d y \\ +\frac{\Delta_{12} f_{1} \varphi^{+}(x, \lambda)}{\omega(\lambda)} \\ \quad \text { for } x \in[a, c)\end{cases}
$$

Consequently,

$$
Y(x, \lambda)=Y_{0}(x, \lambda)+f_{1} \Delta_{12} \frac{\varphi(x, \lambda)}{\omega(\lambda)},
$$

where $G_{0}(x, \lambda)$ and $Y_{0}(x, \lambda)$ are the same with (21) and (22), respectively. From the equalities (13) and (21), it follows that

$$
\left(G_{0}(x, ; \lambda)\right)_{\beta}^{\prime}=\frac{\varphi(x, \lambda)}{\omega(\lambda)}
$$


By using (22), (31), and (33), we deduce that

$$
\begin{aligned}
Y(x, \lambda)= & \Delta_{34} \int_{a}^{c-} G_{0}(x, y ; \lambda) f(y) d y \\
& +\Delta_{12} \int_{c+}^{b} G_{0}(x, y ; \lambda) f(y) d y \\
& +f_{1} \Delta_{12}\left(G_{0}(x, \cdot ; \lambda)\right)_{\beta}^{\prime} .
\end{aligned}
$$

Consequently, the solution $Y(F, \lambda)$ of the operator equation (27) has the form:

$$
Y(F, \lambda)=\left(Y(x, \lambda),(Y(\cdot, \lambda))_{\beta}^{\prime}\right) .
$$

From (34) and (35), it follows that

$$
Y(F, \lambda)=\left(\left\langle G_{x, \lambda}, \bar{F}\right\rangle_{1},\left(\left\langle G_{x, \lambda}, \bar{F}\right\rangle_{1}\right)_{\beta}^{\prime}\right),
$$

where under Green's vector $G_{x, \lambda}$ we mean

$$
G_{x, \lambda}:=\left(G_{0}(x, \cdot ; \lambda),\left(G_{0}(x, \cdot ; \lambda)\right)_{\beta}^{\prime}\right) .
$$

Now, making use of (21), (34), (35), (36), and (37), we see that if $\lambda$ not an eigenvalue of operator $A$, then

$$
\begin{gathered}
Y(F, \lambda) \in D(A), \quad \text { for } F \in H_{1}, \\
Y((\lambda-A) F, \lambda)=F, \quad \text { for } \in D(A), \\
\|Y(F, \lambda)\| \leq|\operatorname{Im} \lambda|^{-1}\|F\|, \quad \text { for } F \in H_{1}, \operatorname{Im} \lambda \neq 0 .
\end{gathered}
$$

Hence, each nonreal $\lambda \in \mathbb{C}$ is a regular point of an operator $A$ and

$$
R(\lambda, A) F=\left(\left\langle G_{x, \lambda}, \bar{F}\right\rangle_{1},\left(\left\langle G_{x, \lambda}, \bar{F}\right\rangle_{1}\right)_{\beta}^{\prime}\right), \quad \text { for } F \in H_{1} .
$$

Because of (38) and (40),

$$
(\lambda-A) D(A)=(\bar{\lambda}-A) D(A)=H_{1}, \quad \text { for } \operatorname{Im} \lambda \neq 0 \text {. }
$$

Theorem 2. The Resolvent operator $R(\lambda, A)$ is compact in the Hilbert space $H_{1}$.

Proof. Let us define the operators $\mathbb{B}_{\lambda}: L_{2}[a, c) \oplus L_{2}(c, b] \rightarrow$ $L_{2}[a, c) \oplus L_{2}(c, b], \widetilde{\mathbb{B}_{\lambda}}: H_{1} \rightarrow H_{1}$ and $\mathscr{C}_{\lambda}: H_{1} \rightarrow H_{1}$ by

$$
\begin{gathered}
\mathbb{B}_{\lambda} f:=\Delta_{34} \int_{a}^{c-} G_{0}(x, y ; \lambda) f(y) d y \\
+\Delta_{12} \int_{c+}^{b} G_{0}(x, y ; \lambda) f(y) d y, \\
\widetilde{\mathbb{B}}_{\lambda} F:=\left(\mathbb{B}_{\lambda} f,\left(\mathbb{B}_{\lambda} f\right)_{b}^{\prime}\right), \\
\mathscr{C}_{\lambda} F:=\left(f_{1} \Delta_{12} \frac{\varphi(x, \lambda)}{\omega(\lambda)}, f_{1} \Delta_{12} \frac{(\varphi(\cdot, \lambda))_{b}^{\prime}}{\omega(\lambda)}\right),
\end{gathered}
$$

respectively. Then we can expressed the resolvent operator $R(\lambda, A)$ as $R(\lambda, A)=\widetilde{\mathbb{B}_{\lambda}}+\mathscr{C}_{\lambda}$. Since the linear operator $\mathbb{B}_{\lambda}$ is compact in the Hilbert space $L_{2}[a, c) \oplus L_{2}(c, b]$, the linear operator $\widetilde{\mathbb{B}_{\lambda}}$ is compact in the Hilbert space $H_{1}$. Compactness $\mathscr{C}_{\lambda}$ in $H_{1}$ is obvious. Therefore, the resolvent operator $R(\lambda, A)$ is also compact in $H_{1}$.

\section{Self-Adjoint Realization of the Problem}

At first, we will prove the following lemmas.

Lemma 3. The domain $D(A)$ is dense in $H_{1}$.

Proof. Suppose that the element $G=\left(g(x), g_{1}\right) \in H_{1}$ is orthogonal to $D(A)$. Denote by $C_{0}^{\infty}[a, c) \oplus C_{0}^{\infty}(c, b]$ the set of infinitely differentiable functions on $[a, c) \cup(c, b]$, each of which vanishes on some neighborhoods of the end-points $x=a, x=c$, and $x=b$. Since $(f(\cdot), 0) \in D(A)$ for $f \in C_{0}^{\infty}[a, c) \oplus C_{0}^{\infty}(c, b]$, we have

$$
\Delta_{34} \int_{a}^{c-} f(x) \overline{g(x)} d x+\Delta_{12} \int_{c+}^{b} f(x) \overline{g(x)} d x=0
$$

for all $f \in C_{0}^{\infty}[a, c) \oplus C_{0}^{\infty}(c, b]$. Since $f$ is arbitrary,

$$
\begin{aligned}
& \int_{a}^{c-} f_{1}(x) \overline{g(x)} d x=0 \\
& \int_{c+}^{b} f_{2}(x) \overline{g(x)} d x=0
\end{aligned}
$$

for all $f_{1} \in C_{0}^{\infty}[a, c]$ and $f_{2} \in C_{0}^{\infty}[c, b]$, respectively. Taking into account that $C_{0}^{\infty}[a, c]$ and $C_{0}^{\infty}[c, b]$ are dense in $L_{2}[a, c]$ and $L_{2}[c, b]$, respectively, we get that the function $g(x)$ is equal to zero as element of $L_{2}[a, c] \oplus L_{2}[c, b]$. By choosing an element $F_{0}=\left(f_{0}(x), T_{b}^{\prime} f_{0}\right)$ such that $T_{b}^{\prime} f_{0}=1$ and putting in $\left\langle F_{0}, G\right\rangle_{1}=0$, we have $g_{1}=0$. So $G=(0,0)$. The proof is completed.

Lemma 4. The linear operator $A$ is symmetric in the Hilbert space $H_{1}$.

Proof. Let $F=\left(f(x), T_{b}^{\prime}(f)\right), G=\left(G_{1}(x), T_{b}^{\prime}(f)\right) \in D(A)$. By partial integration, we get

$$
\begin{aligned}
\langle A F, G\rangle_{1}= & \Delta_{34} \int_{a}^{c-}(\mathscr{L} f)(x) \overline{g(x)} d x \\
& +\Delta_{12} \int_{c+}^{b}(\mathscr{L} f)(x) \overline{g(x)} d x+\frac{\Delta_{12}}{\Delta_{0}} T_{b}(f) \overline{T_{b}^{\prime}(g)} \\
= & \langle F, A G\rangle_{1}+\Delta_{34} W(f, \bar{g} ; c-0)-\Delta_{34} W(f, \bar{g} ; a) \\
& +\Delta_{12} W(f, \bar{g} ; b)-\Delta_{12} W(f, \bar{g} ; c+0) \\
& +\frac{\Delta_{12}}{\Delta_{0}}\left(T_{b}^{\prime}(f) \overline{T_{b}(g)}-T_{b}(f) \overline{T_{b}^{\prime}(g)}\right) .
\end{aligned}
$$

From the definition of domain $D(A)$, we see easily that $W(f, \bar{g} ; a)=0$. The direct calculation gives

$$
\begin{gathered}
T_{b}^{\prime}(f) \overline{T_{b}(g)}-T_{b}(f) \overline{T_{b}^{\prime}(g)}=-\Delta_{0} W(f, \bar{g} ; b), \\
W(f, \bar{g} ; c-0)=\frac{\Delta_{12}}{\Delta_{34}} W(f, \bar{g} ; c+0) .
\end{gathered}
$$


Substituting these equalities in (45), we have

$$
\langle A F, G\rangle_{1}=\langle F, A G\rangle_{1}, \quad \text { for every } F, G \in D(A) \text {; }
$$

so the operator $A$ is symmetric in $H$. The proof is completed.

Remark 5. By Lemma 4, all eigenvalues of the problems (2)-(5) are real. Therefore, it is enough to investigate only real-valued eigenfunctions. Taking in view this fact, we can assume that the eigenfunctions are real-valued.

Corollary 6. If $\lambda_{n}$ and $\lambda_{m}$ are distinct eigenvalues of the problems (2)-(5), then the corresponding eigenfunctions $u_{n}(x)$ and $u_{m}(x)$ are orthogonal in the sense of the following equality:

$$
\begin{gathered}
\Delta_{34} \int_{a}^{c-} u(x) v(x) d x+\Delta_{12} \int_{c+}^{b} u(x) v(x) d x \\
+\frac{\Delta_{12}}{\Delta_{0}} T_{b}^{\prime}(u) T_{b}^{\prime}(v)=0 .
\end{gathered}
$$

Proof. The proof is immediate from the fact that the eigenelements $\left(u(x), T_{b}^{\prime}(u)\right)$ and $\left(v(x), T_{b}^{\prime}(v)\right)$ of the symmetric linear operator $A$ are orthogonal in the Hilbert space $H_{1}$.

Theorem 7. The operator $A$ is self-adjoint in $H_{1}$.

Proof. It is clear that the symmetry of a densely defined $A$ is equivalent to the condition $\langle A F, G\rangle_{1}=\langle F, A G\rangle_{1}$ for all $F, G \in$ $D(A)$. Notice that this implies that $A^{*} \supset A$. If, in addition, we also have that $D\left(A^{*}\right)=D(A)$, then $A$ is self-adjoint. Let $U \in D\left(A^{*}\right)$. Then, by definition of $A^{*}$,

$$
\langle A V, U\rangle_{1}=\left\langle V, A^{*} U\right\rangle_{1}, \quad \forall V \in D(A) .
$$

Let $\lambda_{0}$ be any complex number for which $\operatorname{Im} \lambda_{0} \neq 0$. From this, it follows that

$$
\left\langle\left(\lambda_{0} I-A\right) V, U\right\rangle_{1}=\left\langle V,\left(\overline{\lambda_{0}} I-A^{*}\right) U\right\rangle_{1} .
$$

Since any nonreal complex number is a regular point of $A$, we can define the vector $U_{0} \in D(A)$ as

$$
U_{0}=R\left(\overline{\lambda_{0}}, A\right)\left(\overline{\lambda_{0}} U-A^{*} U\right) .
$$

Hence,

$$
\left(\overline{\lambda_{0}} I-A\right) U_{0}=\overline{\lambda_{0}} U-A^{*} U
$$

Inserting this in (50) and recalling that $A$ is symmetric and $U_{0} \in D(A)$, we have

$$
\begin{aligned}
\left\langle\left(\lambda_{0} I-A\right) V, U\right\rangle_{1} & =\left\langle V,\left(\overline{\lambda_{0}} I-A\right) U_{0}\right\rangle_{1} \\
& =\left\langle V, \overline{\lambda_{0}} U_{0}\right\rangle_{1}-\left\langle V, A U_{0}\right\rangle_{1} \\
& =\left\langle\lambda_{0} V, U_{0}\right\rangle_{1}-\left\langle A V, U_{0}\right\rangle_{1} \\
& =\left\langle\left(\lambda_{0} I-A\right) V, U_{0}\right\rangle_{1} .
\end{aligned}
$$

Consequently,

$$
\left\langle\left(\lambda_{0} I-A\right) V, U-U_{0}\right\rangle_{1}=0 \quad \forall V \in H_{1} .
$$

Since $\lambda_{0}$ is regular point of $A$, we can choose $V=$ $R\left(\lambda_{0}, A\right)\left(U-U_{0}\right)$. Inserting this in the last equality yields $\left\|U-U_{0}\right\|_{1}=0$, and so $U=U_{0}$, and therefore, $U \in D(A)$. The proof is completed.

Remark 8. The main results of this study are derived in modified Hilbert space under simple condition (7). We can show that these conditions cannot be omitted. Indeed, let us consider the next special case of the problems (2)-(5):

$$
\begin{gathered}
-y^{\prime \prime}(x)=\lambda y(x), \quad x \in[-1,0) \cup(0,1], \\
y(-1)=0 \\
(\lambda-1) y^{\prime}(-1)+\lambda y(1)=0 \\
y(0-)=y(0+) \\
y^{\prime}(0-)=-y^{\prime}(0+)
\end{gathered}
$$

for which the condition $(7)$ is not valid $\left(\Delta_{12}<0\right)$. It is easy to verify that the operator $A$ corresponding to this problem is not symmetric in the classic Hilbert space $L_{2}[a, c) \oplus L_{2}(c, b] \oplus$ $\mathbb{C}$ under standard inner-product. Consider the following:

$$
\langle F, G\rangle_{1}:=\int_{a}^{c} f(x) \overline{g(x)} d x+\int_{c}^{b} f(x) \overline{g(x)} d x+\frac{\Delta_{12}}{\Delta_{0}} f_{1} \overline{g_{1}} \text {. }
$$

Moreover, it is well known that the standard Sturm-liouville problems have infinitely many real eigenvalues. But it can be shown by direct calculation that the problem (55) has only one eigenvalue $\lambda=1$.

\section{References}

[1] G. Green, "An essay on the application of mathematical analysis to theories of electricity and magnetism," The Journal für die Reine und Angewandte Mathematik, vol. 39, pp. 73-89, 1850.

[2] C. Neumann, Undersuchungen ber das Logaritmische and Newton'sche Potential, Teubner, Leipzig, Germany, 1877.

[3] E. W. Hobson, "Synthetical solutions in the conduction of heat," Proceedings of the London Mathematical Society, vol. 19, no. 1, pp. 279-294, 1887.

[4] P. Appell, "Sur l'équation $\partial^{2} z / \partial x^{2}-\partial z / \partial y=0$ et la théorie de la chaleur," Journal de Mathématiques Pures et Appliquées, vol. 8, pp. 187-216, 1892.

[5] G. Kirchhoff, "Zur Theorie der Lichtstrahlen," Annalen der Physik, vol. 18, pp. 663-695, 1883.

[6] H. Burkhardt, "Sur les fonctions de Green relatives à un domaine d'une dimension," Bulletin de la Société Mathématique de France, vol. 22, pp. 71-75, 1894.

[7] C. T. Fulton, "Two-point boundary value problems with eigenvalue parameter contained in the boundary conditions," Proceedings of the Royal Society of Edinburgh. A, vol. 77, no. 3-4, pp. 293-308, 1977. 
[8] A. V. Likov and Y. A. Mikhailov, The Heory of Heat and Mass Transfer, Qosenergaizdat, 1963.

[9] I. Titeux and Y. Yakubov, "Completeness of root functions for thermal conduction in a strip with piecewise continuous coefficients," Mathematical Models \& Methods in Applied Sciences, vol. 7, no. 7, pp. 1035-1050, 1997.

[10] J. Ao, J. Sun, and M. Zhang, "The finite spectrum of SturmLiouville problems with transmission conditions," Applied Mathematics and Computation, vol. 218, no. 4, pp. 1166-1173, 2011.

[11] E. Bairamov and E. Uğurlu, "The determinants of dissipative Sturm-Liouville operators with transmission conditions," Mathematical and Computer Modelling, vol. 53, no. 5-6, pp. 805-813, 2011.

[12] B. Chanane, "Sturm-Liouville problems with impulse effects," Applied Mathematics and Computation, vol. 190, no. 1, pp. 610626, 2007.

[13] M. Kadakal and O. Sh. Mukhtarov, "Discontinuous SturmLiouville problems containing eigenparameter in the boundary conditions," Acta Mathematica Sinica (English Series), vol. 22, no. 5, pp. 1519-1528, 2006.

[14] F. S. Muhtarov and K. Aydemir, "Distributions of eigenvalues for Sturm-Liouville problem under jump conditions," Journal of New Results in Science, vol. 1, pp. 81-89, 2012.

[15] O. Sh. Mukhtarov and H. Demir, "Coerciveness of the discontinuous initial-boundary value problem for parabolic equations," Israel Journal of Mathematics, vol. 114, pp. 239-252, 1999.

[16] O. Muhtarov and S. Yakubov, "Problems for ordinary differential equations with transmission conditions," Applicable Analysis, vol. 81, no. 5, pp. 1033-1064, 2002.

[17] M. Shahriari, A. J. Akbarfam, and G. Teschl, "Uniqueness for inverse Sturm-Liouville problems with a finite number of transmission conditions," Journal of Mathematical Analysis and Applications, vol. 395, no. 1, pp. 19-29, 2012.

[18] A. Wang, J. Sun, X. Hao, and S. Yao, "Asymptotic behavior of a differential operator with discontinuities at two points," Mathematical Methods in the Applied Sciences, vol. 34, pp. 373383, 2011.

[19] E. C. Titchmarsh, Eigenfunction Expansions Associated with Second-Order Differential Equations. Part I, Clarendon Press, Oxford, UK, 2nd edition, 1962. 


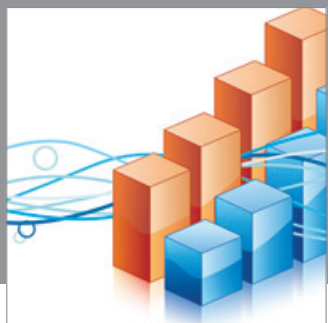

Advances in

Operations Research

mansans

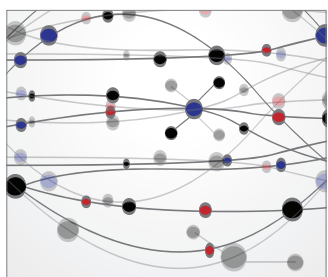

The Scientific World Journal
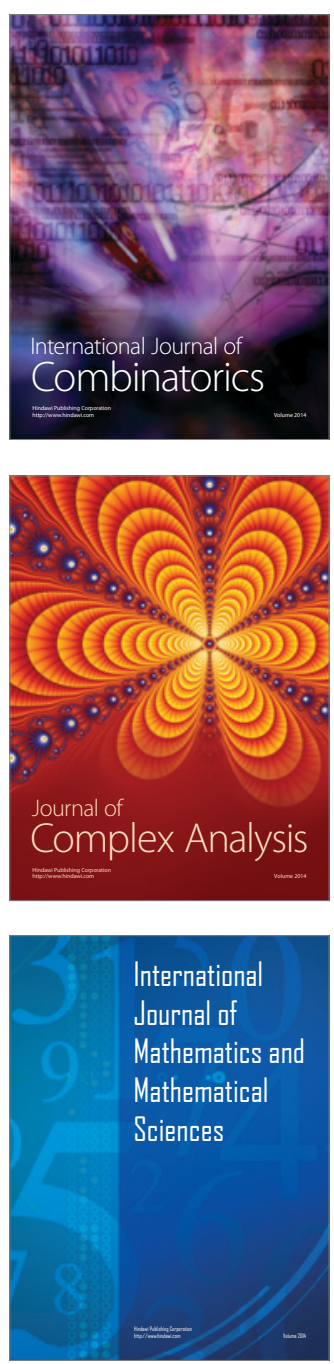
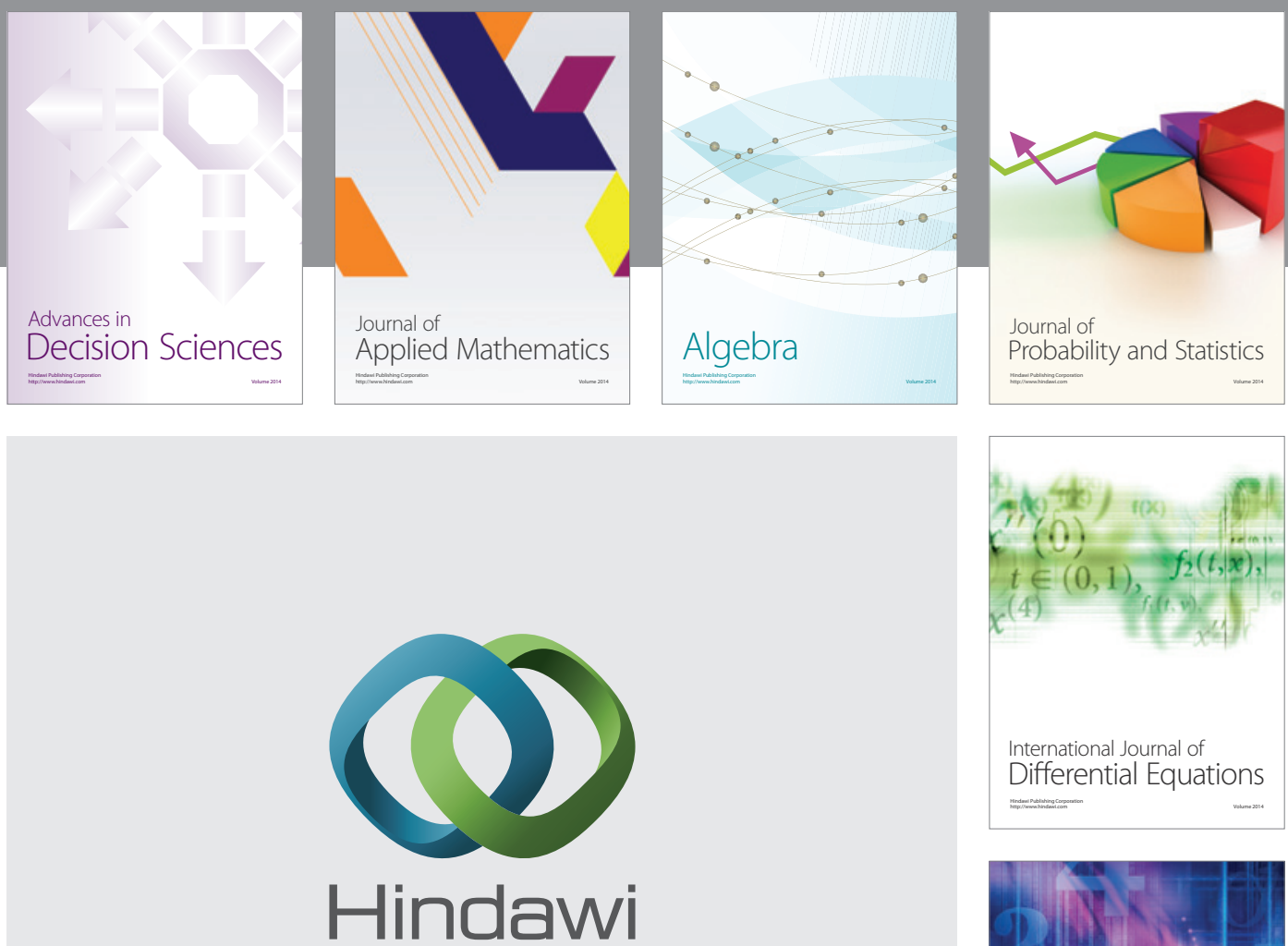

Submit your manuscripts at http://www.hindawi.com
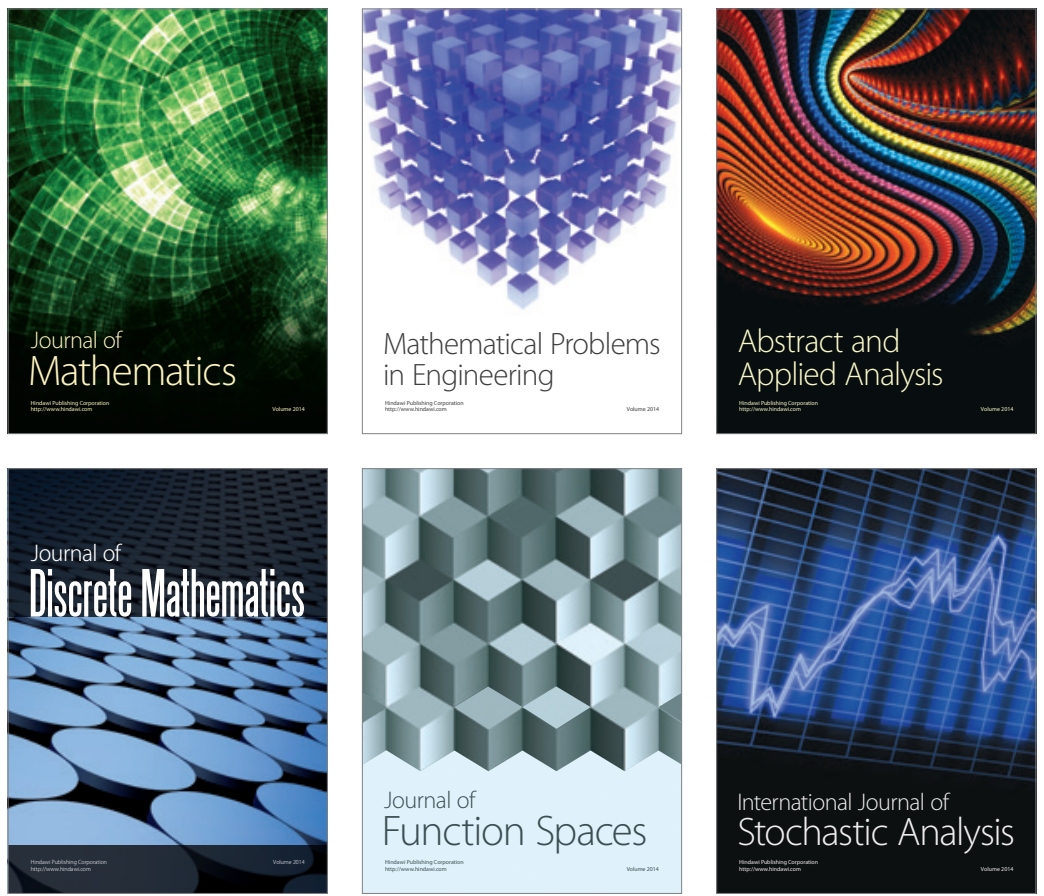

Journal of

Function Spaces

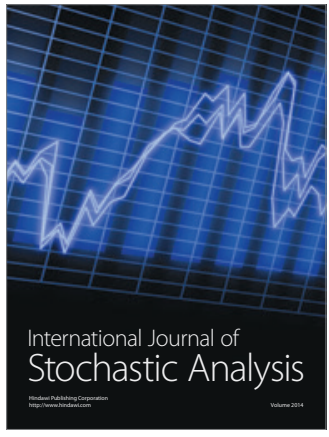

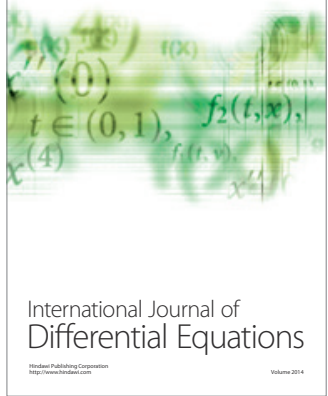
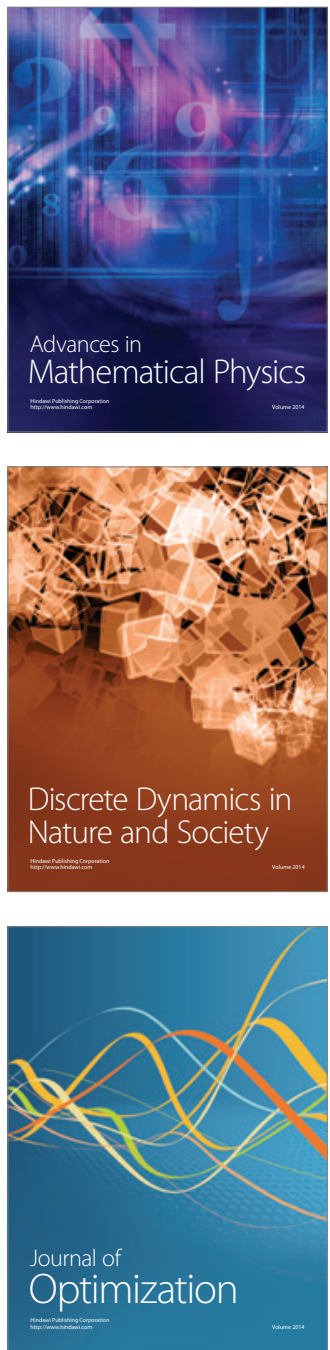